\title{
Evaluación de psicopatología en adultos víctimas de prisión política y tortura durante su infancia y adolescencia
}

MARÍA JOSÉ JORQUERA ${ }^{(1)}$ MICHELLE BRIONES $^{(1)}$ CÉSAR VALENCIA $^{(1)}$ y MARÍA LILIANA CONTRERAS ${ }^{(1)}$ RESUMEN

Objetivo: Evaluar la presencia de indicadores de psicopatología en adultos que fueron prisioneros politicos en infancia y/o adolescencia durante la dictadura militar chilena. Material y método: Estudio de casos y controles en que se compararon indicadores de trastornos mentales en tres grupos: A) adultos con antecedente de tortura en infancia y/o adolescencia sin reparación psicosocial, B) adultos con antecedentes de tortura con reparación psicosocial y C) adultos que han vivido su infancia y/o adolescencia durante la dictadura, sin antecedentes de tortura. Se utilizo una muestra no aleatoria de 60 personas. Se midieron indicadores de depresión, ansiedad (estado/rasgo), trastorno de estrés postraumático (TEPT) y trastorno de personalidad con escala especificas. Resultados: Existen diferencias significativas en los indicadores de trastornos mentales estudiados entre aquellos adultos que tienen antecedentes de tortura y los que no. Sin embargo, no existen diferencias significativas entre aquellos que no han recibido reparación psicosocial de los que si la han recibido. Conclusiones: Existe asociación entre trauma extremo en infancia y/o adolescencia y psicopatología adulta y la posibilidad de cronificarse depende de las variables sociales interactuantes.

Palabras clave: salud mental, tortura.

\section{ABSTRACT}

EVALUATION OF PSYCHOPATHOLOGY EN ADULT VICTIMS OF POLITICAL IMPRISONMENT AND TORTURE DURING THEIR ADOLESCENCE AND CHILDHOOD

Objective: Evaluate the presence of psychopathology indicators in adults who were political prisoners during childhood and/or adolescence, during the Chilean military dictatorship. Materials and methods: Case studies and controls, in which indicators of mental disorders were compared in three groups: A) adults who suffered torture and have not received psychosocial treatment, B) adults who suffered torture who did receive psychosocial treatment, and C) adults whose adolescence and/or childhood took place during the dictatorship, who did not suffer torture. A nonrandom sample of 60 people was used. Indicators of depression, anxiety (state/trait), post traumatic stress syndrome (PTSD) and personality disorder were measured with specific scales. Results: There are significant differences in the indicators of mental disorder studied, between those adults who suffered torture and those that did not. Nevertheless, there are not significant differences between those that received psychosocial treatment and those that did not. Conclusions: There is an association between external trauma in childhood and/or adolescence, and psychopathology in adults, and that the possibility of disorders becoming chronic or not depends on interacting social variables.

Key words: mental health, torture.

(1) Escuela de Psicología. Facultad de Medicina. Universidad de Valparaíso. Avda. Brasil 2140. Valparaíso. Chile. mjjorquerag@hotmail.com 


\section{INTRODUCCIÓN}

Numerosos estudios epidemiológicos señalan que existe una asociación entre trauma infantil y psicopatología en la adultez. Las constelaciones sintomáticas más comunes corresponden a trastornos depresivos, ansiosos, trastorno por estrés postraumático (TEPT) y trastorno de la personalidad (Florenzano y cols (2004) ${ }^{9}$; Vidalis A (2004) ${ }^{17}$; Amir y Lev-Wiesel (2003) ${ }^{1}$; Jonson y cols (1999) ${ }^{13}$ y Robinson $\mathrm{y}$ cols $(1994)^{10}$.

Entre las experiencias infantiles más traumáticas se encuentran las de haber sido objeto de tortura, entendiéndose ésta como "un acto por el cual un grupo de funcionarios públicos o personas que ejerzan funciones públicas inflija intencionadamente sobre otro grupo o una persona dolores o sufrimientos graves, con el fin de obtener información" (CNPPT, 2005) ${ }^{12}$. Se entenderá también como tortura a "la aplicación sobre una persona de métodos tendientes a anular la personalidad de la víctima o a disminuir su capacidad física o mental".

Respecto a lo anterior, en Chile durante la dictadura militar, se torturó con fines políticos a niños(as) y adolescentes. Tras el informe de la Comisión Nacional sobre Prisión Política y Tortura (CNPPT), se estimó que esta singular población corresponde a un $4,3 \%$ del total de personas reconocidas por el Estado, lo que equivale a 1.166 (CNPPT, 2005) $)^{12}$. Si se agregan los 200 adultos que conforman actualmente la Agrupación de Ex Menores Víctimas de Prisión Política y Tortura (2010), pero que no obtuvieron el reconocimiento del Estado, esta cifra ascendería a 1.366 personas, correspondiendo a un 5\%.

En virtud de los antecedentes que aporta la literatura y sumado al hecho de que las 200 personas que constituyen esta Agrupación no han recibido ni reconocimiento ni atención en salud mental por parte del Estado, este trabajo busca estudiar la posible existencia de indicadores de psicopatología en adultos víctimas de prisión política y tortura durante su infancia y adolescencia, en el período histórico de la dictadura militar chilena. De esta forma, se busca contribuir al conocimiento sobre la magnitud de las consecuencias psicológicas de situaciones de represión política, al igual que destacar la importancia de las instancias de reparación para estas personas.

\section{MATERIAL Y MÉTODO}

Corresponde a un estudio transversal, de tipo descriptivo - comparativo, ya que se realizo una sola medición en un momento único (Hernández y cols 1994) ${ }^{11}$.

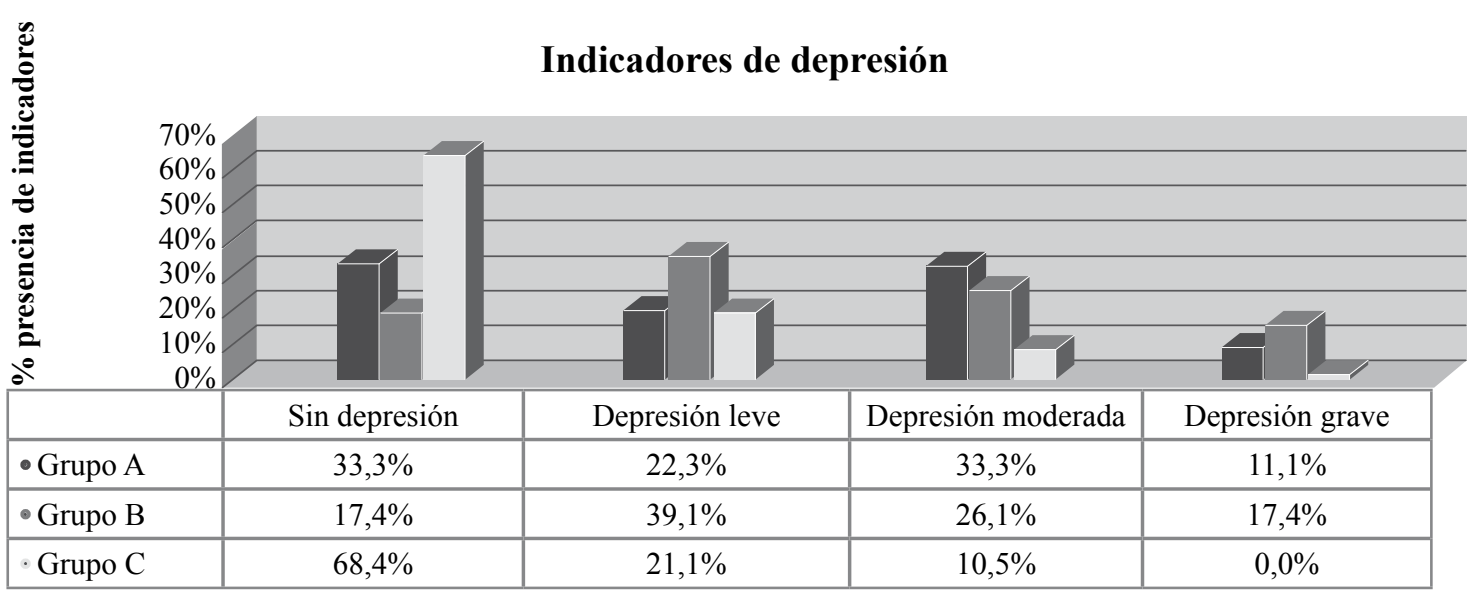

Figura 1. Distribución en porcentajes de Indicadores de depresión según niveles del Inventario de Depresión de Beck. 

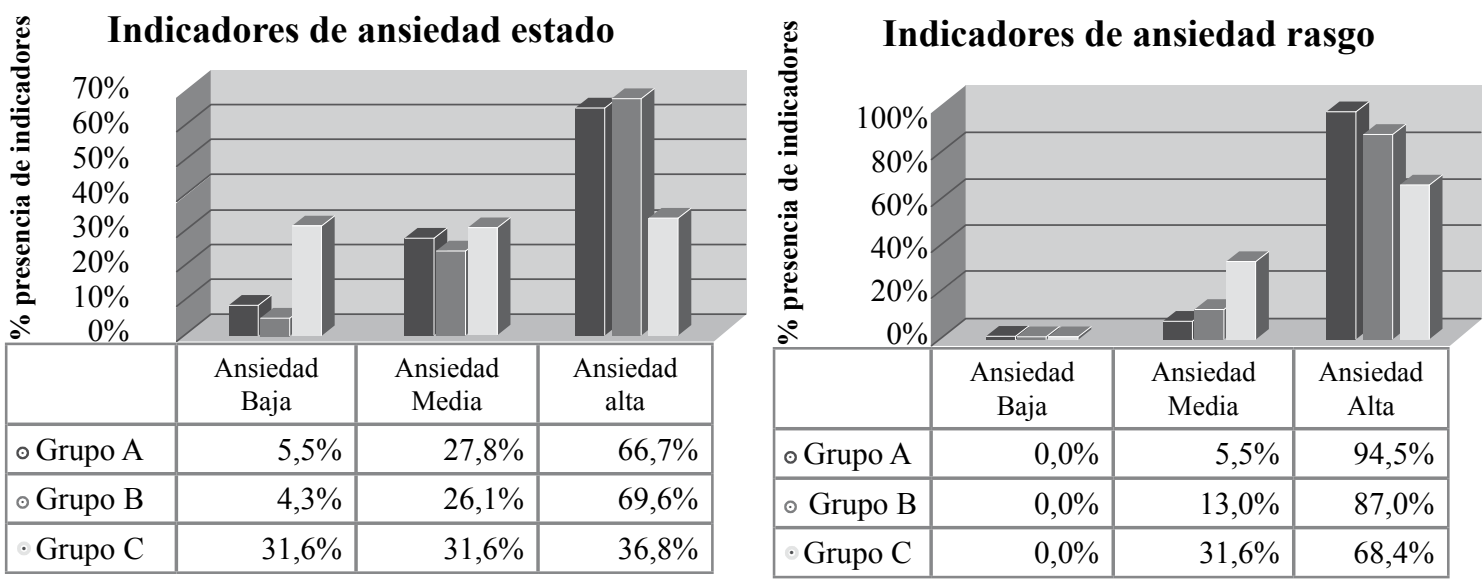

Figura 2. Distribución en porcentajes de indicadores de ansiedad estado y rasgo según niveles del Inventario de Ansiedad Rasgo/Estado de Spielberger.

Para lograr los objetivos propuestos, se obtuvieron 3 muestras con las siguientes características generales:

Grupo A: personas que son ex-menores víctimas de represión política y tortura, que no han recibido atención psicosocial por esta situación.

Grupo B: personas que son ex-menores víctimas de represión política y tortura, y que han recibido atención psicosocial por esta situación.

Grupo C: personas que no son ex-menores víctimas de represión política y tortura.

Las muestras de los grupos A y B se obtuvieron gracias a las dirigentas de la Agrupación de Ex Menores Víctimas de Prisión Política y Tortura, las cuales invitaron a sus integrantes a participar voluntariamente en esta investigación, previa rigurosa revisión del proyecto de estudio. El grupo $\mathrm{C}$ se obtuvo de diversas personas naturales que accedieron voluntariamente a participar (junta de vecinos, auxiliares de colegio etc.), previa encuesta elaborada que igualaba las características sociodemográficas de los grupos A y B.

La composición específica de los tres grupos fue la siguiente:

Grupo A, consta de 18 participantes (30\% muestra total) 7 mujeres y 11 hombres, sus edades van desde los 42 a 50 años, son acreditados por la agrupación y pertenecen a las ba- ses de Antofagasta, Valparaíso, Osorno, Puerto Varas y Fresia, con antecedentes de tortura en infancia y/o adolescencia, y que no han recibido acciones de reparación psicosocial, ya sea proporcionada por el Estado, por la agrupación y/o por vía particular. Sus criterios de exclusión fueron: exiliados, dado que recibieron en el país de asilo político una atención de salud integral; personas que sean atendidas por el programa Prais, y personas reconocidas por el Estado (CNPPT).

Grupo B, consta de 23 participantes (38,8\% muestra total) 17 mujeres y 6 hombres, sus edades van desde los 33 a 54 años, son acreditados por la agrupación y pertenecen a las bases de Antofagasta, Valparaíso, Osorno, Puerto Varas y Fresia, con antecedentes de tortura y que han tenido acceso a reparación psicosocial, ya sea proporcionado por el Estado, por la agrupación o en el país de exilio.

Grupo C, el grupo control, consta de 19 participantes (31,6\% muestra total) 14 mujeres y 5 hombres, sus edades van desde los 30 a 54 años, habitantes de la Provincia de Valparaíso y de Santiago, que vivieron su infancia y/o adolescencia durante el periodo de la dictadura militar, sin antecedentes de tortura. Los criterios de exclusión para este grupo son: que hayan vivido alguna experiencia traumática en su infancia, adolescencia (abuso sexual, abandono, muerte trágica como accidentes o asesina- 
tos de padres o familiar directo, etc.) o adultez (muerte trágica como accidentes o asesinato de hijos, cónyuge o familiar directo) y que tengan familiares víctimas de prisión política y tortura durante la dictadura.

Los indicadores de psicopatología se evaluaron a través de:

- Inventario de Depresión de Beck (BDI) evalúa síntomas depresivos, con mayor porcentaje de ítems cognitivos en consonancia con su teoría (Bobes 2002) .

- Inventario de Ansiedad rasgo/estado de Spielberger (STAI), evalúa ansiedad estado (condición emocional transitoria) y ansiedad rasgo (propensión ansiosa relativamente estable) (Bobes 2002) ${ }^{2}$.

- Escala TOP 8 derivada de la escala de Gravedad del Trastorno por Estrés Postraumático (SI-PTSD) para evaluar indicadores de TEPT (Bobes 2002) ${ }^{2}$.

- Cuestionario IPDE, apartado del DSMIV de la OMS consta de dos secciones: una entrevista semiestructurada y un cuestionario autoadministrado, este último utilizado en el estudio, en el que mediante preguntas de respuesta (verdadero-falso) el paciente describe su conducta habitual durante los últimos 5 años. Este cuestionario de detección proporciona información acerca de qué trastorno/s de la personalidad es probable que esté/n presente/s (Bobes 2002) ${ }^{2}$.

Se realizaron análisis descriptivos para todas las variables que fueron estudiadas y Análisis de Varianza (Anova), seguido de la Prueba de Scheffé para las comparaciones múltiples entre los tres grupos.

\section{RESULTADOS}

En cuanto a indicadores de depresión, en el grupo A el 66,7\% presentó indicadores de sintomatología depresiva, distribuidos de la siguiente forma en sus tres categorías: leve $(22,3 \%)$, moderada $(33,3 \%)$ y grave $(11,1 \%)$; respecto al grupo $\mathrm{B}$, el $82,6 \%$ manifiesta sintomatología depresiva, ya sea leve $(39,1 \%)$, mo- derada $(26,1 \%)$ o grave $(17,4 \%)$; y, finalmente, en el grupo $\mathrm{C}$ el $31,6 \%$ presenta indicadores de depresión, situándose sólo en las categorías leve $(21,1 \%)$ y moderada $(10,5 \%)$ (Figura 1$)$. No hubo diferencias estadísticamente significativas entre los grupos A y B $(p=0,916)$, pero sí las hubo entre ambos y el grupo $\mathrm{C}, \mathrm{p}=0,018$ para la comparación entre el grupo $\mathrm{A}$ y $\mathrm{C}, \mathrm{y} \mathrm{p}$ $=0,003$ para la comparación entre el grupo $\mathrm{B}$ y C).

Para la variable ansiedad-estado, en el grupo A un $66,7 \%$ se ubica en el estado alto, en el grupo B esto se observa en el $69,8 \%$, mientras que sólo un 36,8\% del grupo $\mathrm{C}$ tiene indicadores de ansiedad-estado alto. Respecto a la ansiedad-rasgo, el 94,5\% del grupo A presenta un nivel alto, el $87 \%$ del grupo B se ubica en esta condición, mientras que el $68,4 \%$ del grupo $\mathrm{C}$ se sitúa en dicha categoría (Figura 2). Tanto en "ansiedad-estado" como en "ansiedad-rasgo", los grupos con antecedentes de tortura no muestran diferencias significativa entre sí $(\mathrm{p}=$ 0,975 en ansiedad-estado y ansiedad-rasgo), pero si se encuentran diferencias estadísticamente significativas entre estos dos grupos y el grupo $C(p=0,093$ y $p=0,042$ en ansiedadestado $),(p=0,036$ у $p=0,013$ en ansiedadrasgo).

Respecto del indicador de TEPT, en el grupo A un $83,3 \%$ de los sujetos tiene un indica-

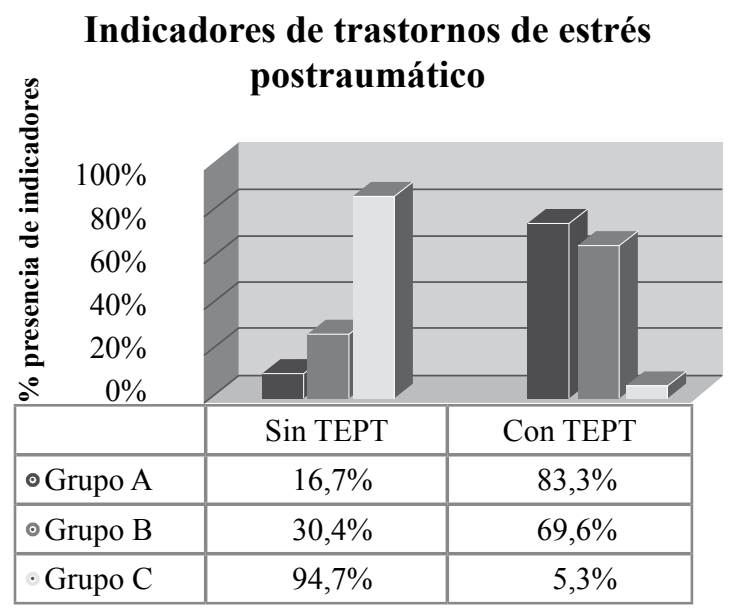

Figura 3. Distribución en porcentajes de indicadores de trastorno categorizados en presencia o ausencia según Escala TOP 8. 


\section{Indicadores de trastorno de personalidad clúster $\mathrm{A}$}

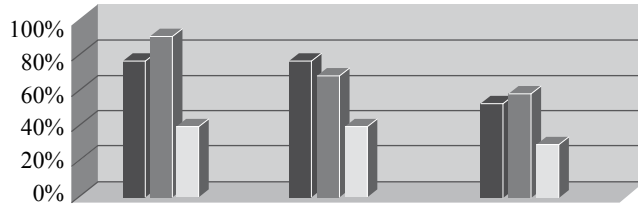

\begin{tabular}{|r|r|r|r|}
\hline & Paranoide & \multicolumn{1}{|c|}{ Esquisoide } & \multicolumn{1}{c|}{ Esquizotipico } \\
\hline - Grupo A & $77,7 \%$ & $72,2 \%$ & $55,5 \%$ \\
\hline - Grupo B & $86,9 \%$ & $65,2 \%$ & $60,8 \%$ \\
\hline Grupo C & $42,1 \%$ & $42,1 \%$ & $26,3 \%$ \\
\hline
\end{tabular}
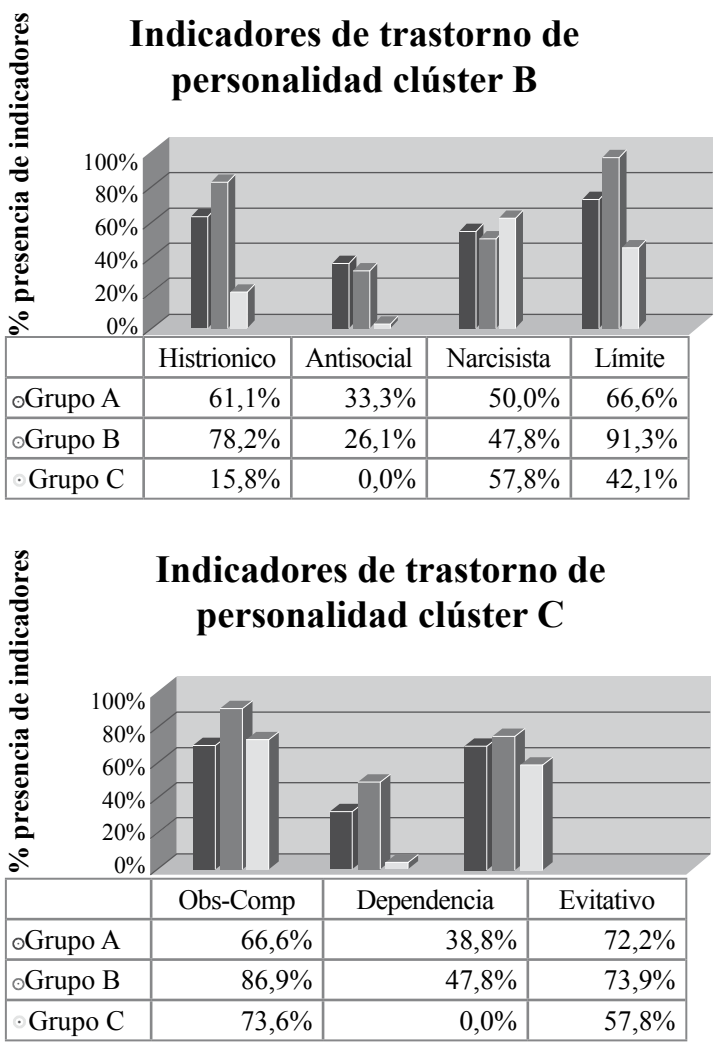

Figura 4. Distribución en porcentajes de participantes con indicadores de trastornos de personalidad según el Examen Internacional de los Trastornos de la Personalidad, IPDE.

dor de sintomatología de TEPT; en el grupo B, esto se presenta en el $69,6 \%$ de los casos, mientras que sólo un 5,3\% del grupo C se sitúa en dicha categoría (Figura 3). Las diferencias no fueron estadísticamente significativas en- tre los grupos con antecedentes de tortura ( $\mathrm{p}$ $=0,980)$, pero si lo fueron entre los grupos $\mathrm{A}$ y C $(p=0,000)$ y entre los grupos B y C $(p=$ $0,000)$.

Con respecto a los resultados sobre indicadores de trastornos de personalidad (Figura 4 y Tabla 1), se observa que para el clúster $\mathrm{A}$, los casos con puntajes de sintomatología en el grupo A fueron distribuidos de la siguiente manera: paranoide $77,7 \%$, esquizoide $72,2 \%$ y ezquizotípico $55,5 \%$; para el grupo B fueron: paranoide $88,9 \%$, esquizoide $65,2 \%$ y ezquizotípico $60,8 \%$; y para el grupo C: paranoide $42,1 \%$, esquizoide $42,1 \%$ y ezquizotípico 25,3\%.

En el caso de los trastornos de personalidad del clúster B, en el grupo A la situación fue la siguiente: histriónico $61,1 \%$, antisocial $33,3 \%$, narcisista $50 \%$ y límite $66,6 \%$; para el grupo B fue: histriónico $78,2 \%$, antisocial $26 \%$, narcisista $47,8 \%$ y límite $91,3 \%$; y para el grupo C los resultados fueron: histriónico $15,8 \%$, antisocial $0 \%$, narcisista $57,8 \%$; y límite $42,1 \%$.

Finalmente, respecto al cluster $\mathrm{C}$ de trastornos de personalidad, el grupo A muestra los siguientes valores: obsesivo compulsivo un $66,6 \%$; dependiente un $38,8 \%$ y evitativo un $72,2 \%$. El grupo B presenta un $86,9 \%$ para obsesivo compulsivo; un $47,8 \%$ para dependiente y un $73,9 \%$ para evitativo. En el grupo C: obsesivo compulsivo un $73,6 \%$; dependiente un $10,5 \%$ y evitativo un $57,8 \%$. Aplicando las pruebas estadísticas se pudo constatar que existen diferencias significativas en la mayoría de los indicadores de trastorno de personalidad de los grupos A y B con el grupo $\mathrm{C}$, en especial en las categorías de paranoide, esquizotípico, histriónico, antisocial, límite y dependiente.

En síntesis, comparando los resultados de los tres grupos en los distintos instrumentos se puede concluir que existen diferencias significativas entre los dos grupos que vivieron situaciones de tortura (grupos A y B) y el grupo control (grupo C). Sin embargo, no hay diferencias significativas entre aquellos que no han recibido atenciones psicosociales (grupo $\mathrm{A}$ ) de los que sí las han recibido (grupo B). 


\section{DISCUSIÓN}

De los puntajes observados, los resultados de los indicadores de depresión, son coherentes con la literatura que asocia trauma en etapa temprana y psicopatología en la adultez, específicamente sintomatología depresiva (Florenzano y cols (2004) ${ }^{9}$; Vidalis A (2004) $)^{17}$. Amir y Lev-Wiesel (2003) ${ }^{1}$; Jonson y cols (1999) ${ }^{13}$ y Robinson y cols $(1994)^{10}$. Cabe recordar el estudio de Amir y Lev-Wiesel (2003)1 que analiza casos de menores expuesto a violencia directa en el periodo del Holocausto, los cuales presentan mayores niveles de depresión en comparación a sobrevivientes que vivieron el mismo acontecimiento en la etapa adulta. J. Barudy $(2009)^{2}$ en su estudio cualitativo con la población estudiada señaló que tal grupo presenta más posibilidades de padecer depresión dadas sus características. Finalmente, considerando el trauma extremo (tortura) como un factor de riesgo para la salud mental, Eitinger $(1995)^{7}$ señala que este tipo de maltrato es un estresor mayor que trae consecuencias a largo plazo e intensifica la tendencia a padecer depresión en edad adulta.

Respecto a los resultados de la variable ansiedad podemos señalar que lo característico de la muestra es que estos adultos se desenvolvieron en un ambiente en el que la amenaza era real y la pérdida de control absoluta. Por ejemplo, la duración y frecuencia de la tortura dependía únicamente del aparato represor generando en la persona un constante estado de alerta y de temor ante eventos futuros o estímulos que se perciben como incontrolables. Lo anterior puede entenderse desde lo planteado por Nemeroff (citado en Valdivia 2006) ${ }^{16} \mathrm{y}$ por Spielberger $(1990)^{15}$, pues las condiciones traumáticas que vivieron estos adultos podrían haberlos predispuesto a reaccionar exageradamente ante el estrés.

De los resultados obtenidos en TEPT podemos concluir que las experiencias de trauma extremo en infancia y/o adolescencia pueden perpetuarse y cronificarse en la etapa adulta. En este caso, el mantenimiento del trauma se debe a que este tipo de acontecimientos son de carácter psicosocial (Estebanez 2005) ${ }^{8}$ es decir, es un fenómeno que afecta a toda una sociedad, pero no en forma uniforme ya que depende de la extracción social y las características personales del individuo. Desde este enfoque, la impunidad -que es una variable social- es un factor mantenedor del trauma individual, dado que imposibilita que la persona pueda diferenciarse a sí mismo como víctima o victimario, teniendo como efecto la dificultad de elaborar la experiencia traumática adecuadamente.

Más allá de la especificidad de los resultados en los trastornos de la personalidad, lo fundamental es señalar que estos adultos desarrollaron gran parte de su proceso evolutivo (infancia y/o adolescencia) en un contexto psicosocial traumático que no corresponde a un proceso evolutivo normativo, en el cual existió una amenaza real a su integridad que sumado a la impunidad constituyeron el escenario habitual en el cual ellos interactuaban. A partir de ese momento posiblemente estas personas adquirieron patrones que si bien respondieron adaptativamente al contexto traumático, hoy dejaron de ser adecuados, ya que las condiciones sociopolíticas cambiaron.

Respecto a la puntuación mayor en los distintos instrumentos de medición en las personas

Tabla 1. Distribución en porcentajes de participantes con indicadores de trastornos de personalidad según el Examen Internacional de los Trastornos de la Personalidad, IPDE.

\begin{tabular}{|l|l|l|l|}
\hline & Grupo A & Grupo B & Grupo C \\
\hline Paranoide & $77,7 \%$ & $86,9 \%$ & $42,1 \%$ \\
\hline Esquizoide & $72,2 \%$ & $65,2 \%$ & $42,1 \%$ \\
\hline Esquizotípico & $55,5 \%$ & $60,8 \%$ & $26,3 \%$ \\
\hline Histriónico & $61,1 \%$ & $78,2 \%$ & $15,8 \%$ \\
\hline Antisocial & $33,3 \%$ & $26,1 \%$ & $0,0 \%$ \\
\hline Narcisista & $50,0 \%$ & $47,8 \%$ & $57,8 \%$ \\
\hline Límite & $66,6 \%$ & $91,3 \%$ & $42,1 \%$ \\
\hline Obs. Comp. & $66,6 \%$ & $86,9 \%$ & $73,6 \%$ \\
\hline Dependencia & $38,8 \%$ & $47,8 \%$ & $10,5 \%$ \\
\hline Evitativo & $72,2 \%$ & $73,9 \%$ & $57,8 \%$ \\
\hline
\end{tabular}


que tienen reparación, ésta puede explicarse a partir de lo que plantea Madariaga (Reflexión 2006) $)^{14}$ a través del concepto de re-traumatización. Las personas que viven las secuelas del terrorismo de Estado, al iniciar un proceso de reparación les implica revivir experiencias dolorosas que por años estaban encapsuladas en la memoria, a esto se suma la tensión de tener que ser escuchado y validado en su relato, lo que no siempre ocurre. De ser aceptada, la persona debe entrar a largas listas de espera para su atención. Por tanto, este proceso de reparación se vive con gran tensión, lo que podría entenderse como una re-experimentación de sus experiencias traumáticas.

Según Keylson (citado en Cintras y cols $2009)^{4}$, es el periodo posterior al hecho traumático inicial (...) lo que determina la perpetuación o no de las secuelas vividas durante el trauma. En el caso chileno consideramos que la impunidad es la prolongación de la represión y, por ende, la perpetuación de una dinámica violenta, pues no permite la integración plena y activa de esta población en la sociedad actual, operando como un factor de riesgo en su salud mental.

También es importante señalar que los traumas en contextos de crímenes de lesa humanidad deben necesariamente ser reparados bajo una visión psicosocial y no sólo a nivel individual (por ej: psicoterapia), ya que la reparación en materia de violación a los derechos humanos implica necesariamente el reconocimiento social y un proceso judicial. En éste ámbito Paz Rojas señala que el aspecto judicial tiene como propósito diferenciar a quienes no han cometido falta de aquellos que si han violado las leyes o reglas, y merecen una sanción (Codepu, 1996) $)^{5}$.

Vinculado a lo anterior es que queremos dejar en claro que este estudio no pretende estigmatizar a los sobrevivientes de la dictadura militar chilena a través de los efectos en su salud mental. Muy por el contrario, queremos destacar el deber y la relevancia de desarrollar acciones de reparación integrales, que incluyan aspectos psicosociales frente a estas situaciones de trauma extremo.
Para finalizar, nuestro estudio revela la potencia de la carga traumática de las primeras generaciones que fueron víctimas directas de la represión política y tortura, y que hoy muchos son padres o madres, pudiendo "heredar" a la segunda generación (posdictadura) un efecto negativo sobre su salud mental, denominado daño transgeneracional, definido éste como la transmisión de la carga traumática a la segunda generación por la imposibilidad de elaborar la experiencia traumática de la primera generación (Cintras y cols 2009) ${ }^{4}$ o, por el contrario, el desarrollo de conductas resilientes.

\section{REFERENCIAS}

1. AMIR M. AND LEV-WIESEL R. (2003) Time Does Not Heal All Wounds: Quality Of Life And Psychological Distress Of People Who Survived The Holocaust As Children 55 Years Later, Journal Of Traumatic Stress, Vol. 16, No. 3. Pp. 295-299.

2. BARUDY J.(2009) Artículo destinado a entregar los fundamentos científicos y éticos de la implicación de los centros EXIL España y EXIL Bélgica (Centro psico-médico-social para refugiados, víctimas de la tortura $\mathrm{u}$ otras violaciones de los derechos humanos) en la reparación terapéutica del daño en niños y niñas víctimas de la tortura durante la dictadura militar en Chile.

3. BOBES, G (2002): Banco de instrumentos básicos para la practica de la psiquiatría clínica. Editorial Ars. Medica Barcelona.

4. CINTRAS,EATIP,GTNM/RJ y SERSOC (2009): Daño Trasgeneracional: consecuencias de la represión política en el cono sur, Santiago de Chile.

5. CODEPU (1996). Persona, Estado, Poder: estudio sobre salud mental. Editorial CODEPU. Santiago de Chile.

6. CONVENCIÓN INTERAMERICANA PARA PREVENIR Y SANCIONAR LA TORTURA (OEA 1998). Art. 2, Promulgada por Chile mediante el decreto $\mathrm{N}^{\circ}$. 809, publicado el 26 de noviembre.

7. EITINGER, LEO. (1995) Prisión en campo de concentración y traumatización psíquica. En: Represión y olvido: efectos psicológicos y sociales de la violencia política dos décadas después. Montevideo, Roca Viva.

8. ESTEBANEZ P. (2005) Medicina Humanitaria. La sociedad ante la guerra: Efectos de la guerra y violencia organizada en la salud mental. Editorial Díaz de Santos. España.

9. FLORENZANO, WEIL, VITRIOL, CRU, CAR- 
VAJAL, FULLERTON y MUÑIZ. (2004) Trauma infanto juvenil y psicopatología adulta: un estudio empírico. Revista Médica de Chile. Volumen 132 $\mathrm{N}^{\circ} 12$, Chile.

10. ROBINSON, S., Y COLS (1994). The present state of people who survived the holocaust as children. Acta Psychiatrica Scansdinavica.

11. HERNÁNDEZ, R. y COLS.(2003) Metodología de la investigación. Editorial Mc GrawHill. México.

12. INFORME DE LA COMISIÓN NACIONAL SOBRE PRISIÓN POLÍTICA Y TORTURA (2005). Editorial Gobierno de Chile. Santiago de Chile.

13. JONSON J. G., PATRICIA COHEN Y COLS.(1999) Childhood maltreatment increases risk for personality disorders during early adulthood. Arch General Psychiatry 56:600-606. Estados Unidos.
14. MADARIAGA C. (2006) Retraumatización: hacia una conceptualización necesaria. En Reflexion 32:48. Santiago de Chile.

15. SPIELBERGER CH. D, DÍAZ-GUERRERO R, STRELAU J.(1990) Cross-cultural anxiety. Editorial ilustrada. Estados Unidos.

16. VALDIVIA MARIO.(2006) Psiquiatría del adolescente. Editorial mediterráneo.

17. VIDALIS A.(2004) Women's Sexual Abuse: 50 Years Later, Psychiatry Journal. Grecia.

Recepción: 3 de marzo de 2011 Aprobación: 8 de junio de 2011

Usted puede comentar éste y otros artículos publicados en la Revista Chilena de Salud Pública, enviando un correo electrónico a revistasp@med.uchile.cl 